Review

\title{
Investigating Molecular Profiles of Ovarian Cancer: An Update on Cancer Stem Cells
}

\author{
Federica Tomao ${ }^{1}$, Anselmo Papa ${ }^{2}$, Martina Strudel ${ }^{2}$, Luigi Rossi², Giuseppe Lo Russo², Pierluigi Benedetti \\ Panici ${ }^{1}$, Francesca Romana Ciabatta ${ }^{2}$, Silverio Tomao ${ }^{2}$
}

1. Department of Gynecology and Obstetrics, Policlinico Umberto I Hospital, University of Rome, Italy;

2. Oncology Unit, ICOT Hospital, Policlinico Umberto I Hospital, University of Rome, Italy.

\begin{abstract}
$\triangle$ Corresponding author: P.zza G. Carbone, 37 - Pontecorvo (FR) - Italy; 320/2231228; anselmo.papa@libero.it.
(c) Ivyspring International Publisher. This is an open-access article distributed under the terms of the Creative Commons License (http://creativecommons.org/ licenses/by-nc-nd/3.0/). Reproduction is permitted for personal, noncommercial use, provided that the article is in whole, unmodified, and properly cited.
\end{abstract}

Received: 2014.01.17; Accepted: 2014.02.09; Published: 2014.03.16

\begin{abstract}
Currently we are more and more improving our knowledge about the characteristics and the role of cancer stem cells in human cancer. Particularly we have realized that self-renewing ovarian cancer stem cells (CSCs) or ovarian cancer-initiating cells, and mesenchymal stem cells (SCs) too, are probably implicated in the etiopathogenesis of epithelial ovarian cancer (EOC). There is clear evidence that these cells are also involved in its intra- and extra-peritoneal diffusion and in the occurrence of chemo-resistance. In assessing the molecular characteristics of ovarian CSCs, we have to take note that these cellular populations are rare and the absence of specific cell surface markers represents a challenge to isolate and identify pure SC populations. In our review, we focused our attention on the molecular characteristics of epithelial ovarian CSCs and on the methods to detect them starting from their biological features. The study of ovarian CSCs is taking on an increasingly important strategic role, mostly for the potential therapeutic application in the next future.
\end{abstract}

Key words: Epithelial ovarian cancer, Ovarian cancer stem cells, Cancer stem cells markers, Tumor microenvironment, Therapeutic targets.

\section{Introduction}

Ovarian cancer is the fifth most common type of cancer in females and the leading cause of mortality for gynecological malignancies, with epithelial carcinoma being the most frequent variety $[1,2]$. EOC is a tumor originating from ovarian epithelial surface $[3,4]$ and it is characterized by epidemiological and clinical features that differentiates it from all other gynecologic malignancies. Within the histology and the pathogenesis of EOC a central role is played by cancer stem cell (CSC), defined as a malignant cancer cell with a stem cell phenotype, according to leading definition proposed in a recent AACR workshop [5]. CSCs support transformation and proliferation of clonal tumor cells favoring their repopulation in the tumor microenvironment. Typically they are predominantly quiescent, have up-regulated DNA repair capacity, are noncommittal to apoptosis and over-express ATP-binding cassette (ABC) drug efflux transporters; moreover they contribute to expanding typical cancer gene signatures $[5,6]$. CSC subpopulation is thought to play a crucial role in tumor development, chemo-resistance and relapse after initial treatment. The first experimental evidences suggesting the existence of CSC came from the study of leukemia patients. Bonnet and co-workers demonstrated that human leukemias are driven by a small population of leukemic stem cells capable of transferring the disease to NOD/SCID mice. This concept was extended to solid epithelial tumors by Al-Hajj and co-workers, who demonstrated that in breast cancer tissues a small population of cells presents stem cell properties, expressing CD24 $4^{\text {low }}$ and CD $44^{\text {high }}$ surface 
markers. Subsequently, CSCs were identified and prospectively isolated from a variety of epithelial cancers, including pancreas, colon and prostate cancers [7-16].

Evaluating their main peculiarities has been shown that CSC has three functional characteristics:

- Transplantability and tumorigenic potential to form tumors when injected into nude mice;

- Specific surface markers;

- Ability to recreate the full phenotypic heterogeneity of the parent tumor $[12,13,17]$.

EOC includes different histological subgroups and therefore all histological cell types have different cellular origin with specific biologic and genetic profiles; many studies can support this theory [18-20]. Consequently, the CSC population for each cancer type may also be variable. It is therefore not surprising that SC properties have been reported in EOC cells isolated using different cell surface markers, including CD44, CD133 or CD24. Each of these EOC cells may represent either a hierarchy of CSC or an entirely different population of CSC for that particular ovarian histology. Moreover, these surface markers are not often specific and this could be a problem for the characterization of CSCs; this is also due to the fact that these cellular populations are rare: isolating and identifying pure SC populations is a real challenge [21-23]. Several signaling pathways, such as sonic Hedgehog, Notch, PTEN, BMI-1, WNT, and p53, are involved in embryogenesis processes. An alteration of these pathways could occur during the development of cancer, leading to dysregulation of SC self-renewal and tumor proliferation [24]. Other pathways involved in carcinogenesis, such as Hedgehog and Wnt, originate from the microenvironment of the SC niche $[25,26]$. This may have very important implications in therapeutic interventions, including explanation for the development of chemo-resistance. There is a critical need to develop broad-spectrum as well as individualized molecular-targeted therapies for EOC; therefore a current crucial research interest is to identify signal transduction pathways and target key molecular role players that direct ovarian tumor sensitivity and resistance to therapy [27]. The aim of this review is to outline recent developments in our understanding of the characteristics of ovarian CSC biomarkers, focusing the interrelationships among selected cells and molecular signal transduction pathways, with the aim to translate these concerns into clinical practice, investigating more active targeted strategies.

\section{Cancer stem cell and tumor prolifera- tion}

Although the mechanisms of malignant trans- formation is not entirely explained by the CSC hypothesis, it has been suggested that CSCs are the malignant counterparts of normal adult tissue SCs, considered unable to carrying on stem cell homeostasis, due to dysregulated signaling pathways. In a work of Valent et al the mechanisms of the malignant transformation were described, showing that the expansion of an initially mutated or epigenetically altered cell (gaining a proliferative advantage over its normal counterparts), leads to the creation of a persistent neoplastic clone. This initial clone may produce distinct subclones and if one or more subclones acquire a particular profile of hits, a malignant tissue develops. At that time, the neoplastic stem cells become CSCs or cancer-initiating cells. The other neoplastic subclones and their stem cells may persist and continue to generate additional pre-malignant and/or malignant subclones [28]. According to this hypothesis, we could say that as well as the normal SCs, also CSCs are thought to reside at the top of the lineage hierarchy and give rise to differentiated cells, which themselves have no potential for self-renewal, and therefore do not contribute significantly to tumor growth. SCs remain in a tissue for longer periods, compared to their differentiated progeny, thereby making them more likely to acquire transforming mutations. Moreover, SCs are more likely to survive to any insults, since they are more resistant to apoptosis and DNA damage [29]. Other mechanisms leading to SC enrichment, under conditions of stress, include heightened DNA damage response and repair, both contributing significantly to tumor survival [30]. A role for CSCs in propagating and maintaining metastases has been proposed [31, 32]. The different hypothesis that the dedifferentiation of mature cells to a more pluripotent state could be a potential mechanism for the development of SC-like features by cancer cells cannot be dismissed.

\section{Regulation of ovarian CSC by tumor microenvironment}

A variety of genes, pathways, and miRNAs are implicated in the regulation of ovarian CSC. In addition, CSC features might be maintained by their surrounding environment, termed the "CSC niche". This niche is a microenvironment composed of heterogeneous populations, including different immune cells, stromal cells, perivascular and vascular cells, tissue macrophages and extracellular matrix components. $[33,34]$

Emerging evidence indicates that the CSC niche is an important condition for sustaining CSC, because signaling from this microenvironment can activate pathways which are essential for the maintenance and function of CSC. More importantly, it has been sug- 
gested that differentiated cells can revert to CSC and, conversely, CSC can differentiate into non-CSC in specific tumor microenvironments, leading to a crucial role of tumor microenvironment in the CSC expression. Several biological processes that occur in the CSC niche, including inflammation, hypoxia and angiogenesis, are deemed capable to determine the fate of CSCs [35].

Several recent studies have reported that hypoxia is one of the key attributes of the tumor microenvironment regulating the properties of ovarian CSC/TICs. For instance, hypoxia increases the survival and chemo-resistance of ovarian CSC/TICs isolated from ovarian cancer cell lines through the induction of HIF-1a mediated c-kit expression. Conversely, hypoxic induction of HIF-1 synthesis can promote the differentiation of ovarian CSCs by inducing TWIST1 expression. A high level of NF-kB activity in ovarian CSCs may be induced by cytokines, such as TNF-a, that are generated as a result of inflammation in the ovarian tumor microenvironment [36].

TGF- $\beta$, that is secreted into the ovarian tumor microenvironment, also affects the growth of ovarian CSCs [37].

Cancer-associated fibroblasts (CAFs) are a type of stromal cells contributing to the constitution of tumor microenvironment and they have demonstrated to promote tumorigenesis by enhancing cell proliferation, angiogenesis, invasion and metastasis [38].

An interesting study demonstrated that the manipulation of miRNAs can convert normal ovarian fibroblasts into ovarian CAFs and vice versa [39].

Recent evidence indicates that CSCs respond to anti-tumour agents differently in vitro and in vivo, reinforcing the concept that the niche in which a CSC is located may be an important determinant of how it responds to a given treatment [40].

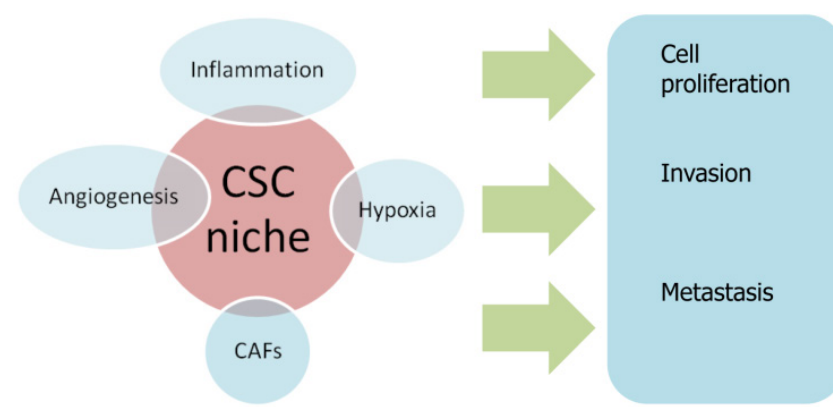

Figure I. Influence of CSC niche on biological processes of cancerogenesis. CAFs: Cancer-associated fibroblasts.

\section{Identification of cancer stem cell}

Several approaches have been described to identify CSCs, including detection of side population (SP) cell phenotype by Hoechst 33342 exclusion, isolation by CSC -specific cell surface marker expression and detection of aldehyde dehydrogenase 1 (ALDH1) activity in the ALDEFLUOR assay $[41,42]$.

\section{I. Detection of Side Population}

Goodell et al. first discovered that display of Hoechst fluorescence, simultaneously at two emission wavelengths, revealed a small and distinct subset of whole bone marrow cells that had phenotypic markers of multi-potential hematopoietic stem cells (HSC).

This subset of cells is referred to as the side population and is enriched for HSCs from murine bone marrow [43]. Many studies of SP have been performed in a number of cancers such as leukemias, brain, prostate, gastrointestinal tract, melanoma, retinoblastoma, and many cancer cell lines, leading to the hypothesis that the SP is enriched with CSC [44-47]. Szotek et al., looking for a possible link between CSCs and chemo-resistance, isolate a SP of cells from genetically engineered mouse ovarian cancer cell lines that expressed the multidrug transporter protein BCRP1 using flow cytometry. They were resistant to doxorubicin. They also isolated a similar smaller SP of cells from the human ovarian cancer cell lines IGROV-1, OVCAR3, and SKOV3, but these SP cells were not further characterized [48].

\subsection{CSC surface markers}

The limitation of using cell surface markers to characterize CSCs is that this approach requires previous knowledge of cell surface markers that are expressed by the putative CSCs in the tissue of interest. Moreover, very often the choice of markers is inferred from known expression of markers in normal adult SCs. CSCs clones have also been identified from tumor ascites; they were able to form anchorage-independent spheroids and have shown to express specific SC markers, as Oct 3/4, Nanog and the progenitor marker Nestin [49]. Latifi et al. identified and profiled the population of ascites cells obtained from ovarian cancer patients, separating adherent (AD) and non-adherent (NAD) cells in culture. AD cells exhibited mesenchymal morphology with an antigen profile of mesenchymal stem cells and fibroblasts. Conversely, NAD cells had an epithelial morphology with enhanced expression of cancer antigen 125 (CA125), EpCAM and cytokeratin 7. They demonstrated a distinct separation of ascites cells into epithelial tumorigenic and mesenchymal non-tumorigenic populations [50]. Several studies have prospectively isolated CSCs by looking for the 
presence of extracellular markers that are thought to be SC specific. The markers most commonly used are CD133 and CD44 [51]. These markers have been used successfully to isolate SCs in normal and tumor tissue [52]. Whilst CD133 and CD44 are thought to be indicative of a CSC phenotype, it is not clear if they are universal markers for characterizing CSCs derived from all types of tumors. Furthermore, expression of CD133 and CD44 may not be restricted to the CSC population and may be present in early progenitor cells. Taken together, all CSC surface markers investigated here are simple indicators, but they cannot be definitely considered reliable markers for defining a population of CSCs in solid tumors since they do not characterize tumor initiating cells exclusively. To increase the sensitivities and specificities in detecting CSCs, further investigations are needed $[48,53]$.

\subsection{CDI33}

The pentaspan transmembrane glycoprotein CD133, also known as Prominin-1, was originally described as a hematopoietic stem cell marker [51]. Subsequently, it was demonstrated that prominin- 1 is commonly expressed by a number of progenitor cells, including epithelial, where it is located on the apical surface [54]. Regarding EOC, Ferrandina G et al. demonstrated that CD133 (+) cells gave rise to a larger number of colonies than those documented in a CD133 (-) population [55]. CD133 ${ }^{+}$ovarian tumor cells were characterized by a higher proliferative potential and clonogenic efficiency than negative cells. For instance CD133 expression, in ovarian carcinoma samples, is related to a poor prognosis, including shorter overall and disease-free survival [56]. The percentages of CD133-1/CD133-2 epitopes expressing cells were significantly lower in normal ovaries/benign tumors with respect to those expressed in ovarian carcinomas. Both the percentages of CD133-1and CD133-2-expressing cells were significantly lower in metastases than in primary ovarian cancer. The authors didn't detect any difference in the distribution of the percentage of CD133-1- and CD133-2-expressing cells according to the clinic-pathologic parameters and the response to primary chemotherapy. Using flow cytometry, Ferrandina G. et al. reported that CD133-1 and CD133-2 were both expressed in human ovarian tumors at higher frequency than in normal ovaries and metastatic omental lesions. CD133-1 and CD133-2 may be useful, therefore, to select and enrich populations of CD133 (+) ovarian tumor cells that are characterized by a higher clonogenic efficiency and proliferative potential [55]. Moreover, in 2009 Baba et al. found that CD133 expression is repressed concurrently with the acquisition of DNA methylation in CD133- progeny of
CD133+ cells, supporting a role for CD133 in CD133+ cells, which is not required in CD133- cells after asymmetric division [57]. Expression of CD133-1 and CD133-2, which were detected in ovarian carcinomas, was also observed in normal ovaries. CD133- cells from cancer cell lines, primary tumors and ascitic ovarian fluid, were shown to be also tumorigenic. $\mathrm{CD}_{133}{ }^{+}$cells, derived from ovarian tumors, were capable of self-renewal and were associated with increased tumor aggression in xenografts [55, 57]. According to these discoveries, Curley et al. demonstrated that tumor-derived CD133-1 cells have an increased tumorigenic capacity and are capable of recapitulating the original heterogeneous tumor [58].

\subsubsection{CD44}

CD44 is a surface molecule which mediates cell adhesion and migration by binding extracellular matrix components such as hyaluronic acid, osteopontin, or activating receptor tyrosine kinases, which are related to tumor progression and metastatic progression $[7,59]$. CD44 is involved in cell-cell interactions, cell adhesion and migration, but it constitutes also a receptor for hyaluronic acid, activating a variety of receptor tyrosine kinases in many cancer types. According to this role, it drives some mechanisms favoring an increase in the proliferation and survival rates of tumor cells, by the activation of the MAPK and PI3K/AKT pathways [60, 61].

CD44 expression has been associated with poor prognosis and resistance to chemotherapy. CD44 positive cells have been shown to express high levels of other stem cell markers, such as Oct4 and nestin. Moreover, CD44 enhance NFKb activity and inflammatory cytokine effects, including high expression of IL1b, IL6, and IL8. These CD44-mediated characteristics could influence the response of patients to chemotherapy, resulting in negative prognosis. $[62,63]$ Bapat et al. found that the growth factor receptors c-met and EGFr were up-regulated in ovarian CSCs as well as CD44, expressing also E-cadherin. Correspondingly, Snail, a known mediator of EMT through transcriptional repression of E-cadherin, was expressed in some CSC clones and to a lesser extent in others [64]. Chen et al. demonstrated in vitro that human epithelial ovarian cancer CD44+/CD117+ cells have the properties to make the tumor be chemoresistant to conventional therapies, such as 5FU, docetaxel, cisplatin, and carboplatin [65]. CD44 has also been demonstrated to be associated with other CSC markers. In fact, Wei at al., investigating about Müllerian Inhibiting Factor with the aim to inhibit stem progenitors in EOC, identified eight marker panels on three human ovarian cancer cell lines and found that the combination of Epcam ${ }^{+}, \mathrm{CD}_{2}{ }^{+}$, and 
$\mathrm{CD} 44^{+}$formed more colonies than other marker combinations. It was necessary to use these $3+$ panels in combination, as each marker alone was not sufficiently selective [66]. Two studies have independently defined ovarian cancer SC by evaluating CD44+ CD117+ and CD133+ phenotypes. The latter suggests an epigenetic regulation of the CD133 promoter [64, 67]. Additionally, using CD44, stem-like cells were enriched from patients' samples and were characterized by Myd 88 expression and chemokine and cytokine production [68]. It is likely that both CD133 and CD44 expression characterize ovarian CSC. Alternatively, there may be more than one population of cells with SC properties in ovarian cancers. Generally, these studies highlight the lack of consensus about the molecular characteristics of ovarian CSCs.

\subsubsection{CD24}

CD24 is a glycosylphosphatidylinositol-linked cell surface protein expressed in various solid tumors [69]. Expression of CD24 represented a marker of poor prognosis in ovarian cancer. A study demonstrated that CD24 could localize in the cytoplasm of ovarian serous tumors, while normal epithelium and serous cystadenomas expressed CD24 marker in the apical membrane. Thus, the cytoplasmic expression of CD24 could be used as a specific marker to predict survival rates and recurrence of cancer [70, 71]. Gao et al. have successfully isolated CD24+ CSCs from ovarian tumor specimens and identified CD24 as a putative CSC marker in EOC [72]. In this study, CD24 cells were shown to proliferate slowly, were more resistant to chemotherapy, and demonstrated enhanced tumorigenicity potential compared to CD24- cells. The depletion and over-expression of CD24 could regulate the phosphorylation of STAT3 and FAK by affecting Src (non-receptor tyrosine kinases) activity.

\subsection{4. $\mathrm{CD} I 17$}

CD117, known as c-kit, is a type III receptor tyrosine kinase involved in cell signal transduction. It is involved in various cellular processes, including apoptosis, cell differentiation, proliferation, and cell adhesion [64]. High expression level of CD117 was observed in ovarian cancers [48]. Luo and his colleagues further demonstrated that CD117+ ovarian cancer cells had the ability to self-renew, differentiate, and regenerate tumors compared to CD117- in xenograft model [69]. It has been also suggested that CD117 in ovarian carcinoma was associated with poor response to chemotherapy [73]. The activation of Wnt/ $\beta$-catenin-ATP-binding cassette G2 pathway was required for cisplatin/paclitaxel-based chemoresistance caused by CD117 in ovarian CSCs [74].

\subsection{5. ЕрСAM}

The epithelial cell adhesion molecule EpCAM is a glycosylated membrane protein expressed in different solid tumors, including colon, lung, pancreas, breast, head and neck and ovary [75]. EpCAM was found to be hyperglycosylated and frequently associated with cytoplasmic staining in carcinoma tissues [73]. It is comprised of an extracellular domain (EpEX), a single transmembrane domain and a short 26-amino acid intracellular domain (EpICD). Among them, EpEX is involved in cell-cell adhesion [74]. In fact, down-regulation of EpCAM could cause the loss of cell-cell adhesion and promote epithelial- mesenkymal transformation (EMT), a step toward tumorigenensis [75,76]. Metastatic and recurrent tumors were found to express significantly higher levels of EpCAM when compared with primary carcinomas. EpCAM expressing cells have also been described to possess a tumor-initiating role with stem/progenitorlike features [77].

\subsection{ALDHIAI and ALDEFLUOR assay}

A valid marker detected in several malignant and normal tissues is aldehyde dehydrogenase-1A1 (ALDH1A1). ALDH1A1 is an intracellular enzyme that participates in cellular detoxification, differentiation, drug resistance, through the oxidation of intracellular aldehydes, and management of the differentiation pathways. It is not only a stemness marker, but it also play an important role in the biology of tumor initiating cells [78]. ALDH1A1 was associated with chemo-resistance in the ovarian CSC too [79]. It has been demonstrated that mouse/human hematopoietic/neural stem and progenitor cells have high ALDH1 activity. High ALDH1 activity, associated with poor clinical outcome, has been reported in breast cancer cells, ovarian cancer cells and glioblastomas. Therefore, the use of ALDH1 activity, as a purification strategy, allows the non-toxic and efficient isolation of human stem-like cells, based on a conserved stem/progenitor cell function [80,81]. ALDH has been also studied in association with CD133 in order to identify a set of markers to identify ovarian CSCs. Siva et al. discovered that the presence of ALDH $(+) C D 133(+)$ cells in debulked primary tumor specimens was correlated with reduced disease-free and overall survival in ovarian cancer patients [82]. ALDH activity is commonly detected using an ALDEFLUOR assay. Cells are stained by bodipyaminoacetaldehyde (BAAA) at $1.5 \mathrm{mM}$ and incubated for $30 \mathrm{~min}$ at $37 \mathrm{uC}$. An inhibitor of ALDH1, diethylamino- benzaldehyde (DEAB), was used as a negative control at a10-fold molar excess [83]. 


\section{Signaling pathways as potential targets for CSCs}

Tumorigenic and self-renewal features of CSC are also conditioned by Wnt, Sonic Hedgehog (Shh), and Notch signaling [84].

Wnt signaling plays a key role in the embryonic development of the ovary, and it is involved in normal follicular development and ovarian function. In ovarian cancer its aberrant regulation is implicated in cancer development [85]. One of the most common Wnt and Hedgehog target genes, leucinerich G protein-coupled receptor 5 (Lgr5, also known as Gpr49), has been shown to mark rapidly cycling stem cells in the small intestine and hair follicles [86]. Lgr5 is an orphan seven-transmembrane domain receptor with similarity to thyroid-stimulating hormone, follicle-stimulating hormone and luteinizing hormone receptors. Selective up regulation of Lgr5 has been reported in ovarian, colon and hepatocellular carcinomas [87].

Recently an activation of the STAT3 pathway in ovarian tumors has been reported. Hence, targeting STAT3 signaling pathways in CSCs of ovarian tumors may represent a novel approach to overcome CSC-mediated chemo-resistance [88]. A recent study has shown that the silencing of jagged 1, a Notch ligand, could sensitize ovarian cancer cell lines to taxane through cross talk with the Hedgehog pathway, suggesting that the Hedgehog pathway represents an important target to eradicate ovarian tumorigenesis [89].

\section{Chemoresistance and CSC target ther- apy}

The CSC theory supports that it's sufficient a small residual number of CSCs in situ after treatment, so that disease recurrence can occur [90]. Although the standard combination of surgery and chemotherapy can effectively reduce tumor mass, most patients, eventually with residual ovarian CSCs, acquire chemo-resistance [91, 92]. Several pathways could be involved in these mechanisms including activation of anti-apoptotic factors, inactivation of pro-apoptotic effectors, and/or reinforcement of survival signals [93]. The aim to test alternative models of treatment, in order to overcome the clinical problem of resistance, lead to the discovery and evaluation of several novel molecular targeted agents directed against the molecular pathways we described above, involved in ovarian carcinogenesis. In this context the study of ovarian cancer stem cells is taking on an increasingly important strategic role, mostly for the potential therapeutic application in next future [94].

\section{I. Resistance to conventional therapy}

Based on an understanding of their characteristics, the refractory response of CSCs to drugs and radiation treatments may be attributed to:

- Drug effluxion: some of most frequently studied drug transporters, associated with acquisition of resistance in normal SCs as well as in CSCs, are multifunctional efflux transporters from the $A B C$ gene family [95]. These contribute to tumor resistance by actively transporting drugs across cell membranes through ATP hydrolysis [96].

- Glutathione (GSH) system: the GSH system can suppress oxidative stress and maintain cellular redox homeostasis [97]. The contribution of GSH and GSH-related enzymes to chemo-resistance has been demonstrated in different types of tumor, including ovarian cancer and brain tumor [98]. In a recent study, it has been shown that platinum-resistant human cancer cells with stem-cell like properties, had high cellular GSH and accumulated significantly less cellular platinum compared to their parental cells. Moreover they failed to undergo apoptosis when exposed to platinum at drug concentrations toxic to the parental cells [99].

- Apoptosis: apoptosis can condition response to antitumor drugs and it's regulated by several molecular phenomena, such as the expression of Bm- 1 and the loss of p53.

- Enrichment of CSCs during disease progression: enrichment of CSCs in tumor tissues is reported in patients with response to therapy, through mechanisms such as enhanced DNA damage repair and changes in the cellular phenotype between epithelial and mesenchymal states of cell [100]. Epithelial to mesenchymal transition (EMT) is a physiological transcriptional reprogramming event and is characterized by the combined loss of epithelial cell junctions and cell polarity and the gain of a mesenchymal phenotype. EMT and mesenchymal to epithelial transition (MET) processes are now well recognized in cancer progression [101]. A link between CSC and EMT has been suggested, whereby transformed human mammary epithelial cells, that have undergone EMT, show a gain of the CSC phenotype [102].

- tumor dormancy and CSC quiescence: many CSCs stay in G0 and are not susceptible to cell cycle-specific chemotherapeutic agents [103]. Consequently, this sub-population could survive to such treatments, later becoming able to regenerate the tumor [104]. 


\subsection{Target therapy}

Antibody therapies against tumor cell surface antigens have improved clinical prognosis through inhibition of specific signaling pathways, enhancing activation of direct immune effectors. In some cases, antibodies are conjugated with a bioactive drug that enables selective targeting of chemotherapeutic agents. In other cases, they block a signaling pathway in which the marker may be involved. A major goal in CSC research is to devise new strategies that will kill CSCs. To date, most efforts have focused on searches for targets expressed exclusively in CSCs, or on screens for selective drugs or RNA interference (RNAi) molecules that selectively kill CSCs, but not normal stem cells. Candidate therapeutic targets include various receptors and their oncogenic derivatives, adhesion molecules, antibody-accessible surface components, signaling intermediates, survival pathway elements, chromatin modifiers and metabolic targets. Gene expression profiling, next-generation sequencing and increasingly sophisticated screening methods are anticipated [105-125] (Table 1).

Table I. Agents targeting ovarian CSC in vitro and in vivo.

\begin{tabular}{|c|c|c|c|c|}
\hline Agent & Target & Effects & Mechanism of action & Reference \\
\hline Niclosamide & $\begin{array}{l}\text { Metabolic pathways in Side } \\
\text { Population }\end{array}$ & Increased sensitivity to cisplatin & Depletion of CSC & Yo et al. [105] \\
\hline Isoflavane derivate & $\begin{array}{l}\text { Mitochondria in } \\
\text { CD44+MyD88+ cells }\end{array}$ & $\begin{array}{l}\text { Induction of apoptosis in ovarian } \\
\text { CSC in vitro }\end{array}$ & $\begin{array}{l}\text { Inhibition of mitochondrial } \\
\text { function }\end{array}$ & Alvero et al. [106] \\
\hline Eriocalyxin B & $\begin{array}{l}\text { NF-kB pathway in CD } 44+ \\
\text { cells }\end{array}$ & $\begin{array}{l}\text { Induction of apoptosis in ovarian } \\
\text { CSC in vitro }\end{array}$ & Inhibition of NF-kB pathway & Leizer et al. [107] \\
\hline Metformin & ALDH+ cells & $\begin{array}{l}\text { Inhibition of ovarian CSC in vitro } \\
\text { and in vivo }\end{array}$ & $\begin{array}{l}\text { Depletion of CSC and inhi- } \\
\text { bition of the formation of } \\
\text { CSC spheres }\end{array}$ & Shank et al. [108] \\
\hline $\begin{array}{l}\text { Small hyaluronan oli- } \\
\text { gosaccharides }\end{array}$ & $\begin{array}{l}\text { Hyaluronan-CD } 44 \text { interac- } \\
\text { tion in CD133+ cells }\end{array}$ & $\begin{array}{l}\text { Inhibition of growth of EOC with } \\
\text { high levels of CD133 in vivo }\end{array}$ & $\begin{array}{l}\text { Inhibition of the hyalu- } \\
\text { ronan-CD44 interaction }\end{array}$ & $\begin{array}{l}\text { Slomiany et al. } \\
\text { [109] }\end{array}$ \\
\hline $\begin{array}{l}\text { Clostridium perfringes } \\
\text { enterotoxin (CPE) }\end{array}$ & Claudin-4 in CD44+ cells & $\begin{array}{l}\text { Inhibition of growth of tumor in } \\
\text { chemotherapy-resistant CD } 44+ \\
\text { CSC in vivo }\end{array}$ & CPE-induced cytotoxicity & $\begin{array}{l}\text { Casagrande at al. } \\
{[110]}\end{array}$ \\
\hline$\gamma$-secretase inhibitors & $\begin{array}{l}\text { Notch pathway in Side Pop- } \\
\text { ulation }\end{array}$ & Increased sensitivity to Cisplatin & Depletion of ovarian CSC & $\begin{array}{l}\text { McAuliffe et al. } \\
\text { [111] }\end{array}$ \\
\hline
\end{tabular}

\section{Discussion}

Ovarian CSCs are likely to be heterogeneous as well as the EOC itself. We can discover this heterogeneity not only in tumors of the same kind in different patients, but also in different cancer cell populations of a single patient. As well as other human malignancies, EOC evolution and heterogeneity could be explained by the CSC model. This feature is due to the fact that the carcinogenesis process goes on basing on the development of subclones with different features and different control of many properties. Investigation of tumorigenic cells should be conducted in the light of recent results demonstrating that populations responsible for driving tumor progression are actually in a transient phase of altered cell cycling, gene expression and drug sensitivity. The existence of a CSC population is confirmed by the experimental finding that a small and distinguishable group of malignant cells can generate a cancer when transferred into immune-deficient mice. Then, the key role of CSCs is clear, and these cells are beginning to represent a possible target, because their elimination could contrast the tumor growth leading to a sustained disease-free outcome or even definitive cure in many patients. If such outcome could be reached in the management of EOC, it would represent a completely new approach to a disease that is still linked to high mortality even in early diagnosed patients.

The first step to proceed towards this therapeutic strategy is the correct identification of CSCs and this is a process that nowadays still presents many difficulties. As described above, the CSCs can be identified and isolated by different methodologies, including isolation and detection of side population phenotype by Hoechst 33342 exclusion, CSC-specific cell surface marker expression, and ALDH activity assay. None of the methods mentioned are exclusively used to isolate the solid tumor CSCs, highlighting the imperative to delineate more specific markers or to use combinatorial markers and methodologies. Until now, no universal single marker has been found to faithfully isolate ovarian CSCs. We can say that, even in multi-passaged cancer cell lines, hierarchic government of growth and differentiation is conserved and that the key CSC population may be composed of small overlapping cell fractions defined by various arbitrary markers. CSC marker expression is not static but con- 
stantly shifting, during differentiation or continuously as an environmental response. Moreover CSC markers are often being connected to the CSC properties of drug resistance, proliferation, self-renewal and metastasis. Increased precision in the details of the methods used and described in identifying CSCs, using all of these markers, will be central for the progress of knowledge in this field.

In this context, EOC chemo-resistance may be attributable to the fact that, during its development, the tumor is more and more enriched of CSC, whose resistance to conventional therapies is linked to the different mechanisms we described above. Although these markers are important for defining aggressive and resistant cell populations, it is also necessary to investigate other useful strategies, mainly targeting these surface proteins with blocking antibodies to inhibit tumor progression.

CSC characterization and identification could really represent the key process towards a better management of EOC and other malignancies, leading to a more accurate disease definition and to a tailored therapeutic approach in the next future.

\section{Competing Interests}

The authors have declared that no competing interest exists.

\section{References}

1. Siegel R, Ward E, Brawley O, et al. Cancer statistics, 2011. CA Cancer J Clin. 2011;61:212-236

2. Wang L, Mezencev R, Bowen NJ, et al. Isolation and characterization of stem-like cells from a human ovarian cancer cell line. Mol Cell Biochem. 2012;363:257-268.

3. Murdoch WJ, McDonnel AC. Roles of the ovarian surface epithelium in ovulation and carcinogenesis. Reproduction. 2002;123(6):743-750.

4. Ness RB, Cottreau C. Possible role of ovarian epithelial inflammation in ovarian cancer. J Natl Cancer Inst 1999, 91(17):1459-1467.

5. Clarke MF, Dick JE, Dirks PB, et al. Cancer stem cells-perspectives on current status and future directions: AACR Workshop on cancer stem cells. Cancer Res. 2006;66:9339-9344.

6. Pardal R, Clarke MF, Morrison SJ: Applying the principles of stem-cell biology to cancer. Nat Rev Cancer. 2003;3:895-902.

7. Al-Hajj M, Wicha MS, Benito-Hernandez A, et al. Prospective identification of tumorigenic breast cancer cells. Proc Natl Acad Sci U S A. 2003;100:3983-3988.

8. Galli R, Binda E, Orfanelli $U$, et al. Isolation and characterization of tumorigenic, stem-like neural precursors from human glioblastoma. Cancer Res. 2007;64:7011-7021.

9. O'Brien CA, Pollett A, Gallinger S, et al. A human colon cancer cell capable of initiating tumour growth in immunodeficient mice. Nature. 2007:445:106-110.

10. Prince ME, Sivanandan R, Kaczorowski A, et al. Identification of a subpopulation of cells with cancer stem cell properties in head and neck squamous cell carcinoma. Proc Natl Acad Sci U S A. 2007;104:973-978.

11. Li C, Heidt DG, Dalerba P, et al. Identification of pancreatic cancer stem cells. Cancer Res. 2007;67:1030-1037.

12. Quintana E, Shackleton M, Sabel MS, et al. Efficient tumour formation by single human melanoma cells. Nature. 2008;456(7222):593-598.

13. Bonnet D, Dick JE: Human acute myeloid leukemia is organized as a hierarchy that originates from a primitive hematopoietic cell. Nat Med.1997;3:730-737.

14. Tomao F, Papa A, Rossi L, et al. Emerging role of cancer stem cells in the biology and treatment of ovarian cancer: basic knowledge and therapeutic possibilities for an innovative approach. J Exp Clin Cancer Res. 2013;32:48. doi: 10.1186/1756-9966-32-48.

15. Collins AT, Berry PA, Hyde C, et al. Prospective identification of tumorigenic prostate cancer stem cells. Cancer Res. 2005;65:10946-10951.

16. Gao MQ, Choi YP, Kang S, et al. CD24+ cells from hierarchically organized ovarian cancer are enriched in cancer stem cells. Oncogene. 2010;29:2672-2680.
17. Dalerba P, Clarke MF: Cancer stem cells and tumor metastasis: first steps into uncharted territory. Cell Stem Cell. 2007;1:241-242.

18. McCluggage WG. Morphological subtypes of ovarian carcinoma: a review with emphasis on new developments and pathogenesis. Pathology. 2011;43(5):420-32.

19. Lim D, Oliva E. Precursors and pathogenesis of ovarian carcinoma. Pathology. 2013;45(3):229-42.

20. Auersperg $\mathrm{N}$. The origin of ovarian carcinomas: a unifying hypothesis. Int J Gynecol Pathol. 2011;30(1):12-21.

21. Hill RP. Identifying cancer stem cells in solid tumors: case not proven. Cancer Res. 2006;66:1891-1895.

22. Vogel G: Stem cells. 'Stemness' genes still elusive. Science. 2003;302:371.

23. Zon LI: Intrinsic and extrinsic control of haematopoietic stem-cell self-renewal. Nature. 2008;453:306-313.

24. Reya T, Morrison SJ, Clarke MF, et al. Stem cells, cancer, and cancer stem cells. Nature. 2001;414:105-111.

25. Liu S, Dontu G, Mantle ID, et al. Hedgehog signaling and Bmi-1 regulate self-renewal of normal and malignant human mammary stem cells. Cancer Res. 2006;66(12):6063-6071.

26. Korkaya H, Paulson A, Charafe-Jauffret E, et al. Regulation of mammary stem/progenitor cells by PTEN/Akt/ $\beta$-catenin signaling. PLoS Biol. 2009;7(6):e1000121

27. Itamochi H. Targeted therapies in epithelial ovarian cancer: molecular mechanisms of action. World Journal of Biological Chemistry. 2010;1(7):209-220.

28. Valent $\mathrm{P}$, Bonnet $\mathrm{D}$, De Maria R, et al. Cancer stem cell definitions and terminology: the devil is in the details. Nature Rev. Cancer. 2012;12:767-775.

29. Croker AK, Allan AL. Cancer stem cells: implications for the progression and treatment of metastatic disease. J Cell Mol Med. 2008;12(2):374-390.

30. Frosina G. DNA repair in normal and cancer stem cells, with special reference to the central nervous system. Curr Med Chem. 2009;16:854-866.

31. Charafe-Jauffret E, Ginestier C, Iovino F, et al. Breast cancer cell lines contain functional cancer stem cells with metastatic capacity and a distinct molecular signature. Cancer Res. 2009;69(4):1302-1313.

32. Dontu $G$, Abdallah WM, et al. In vitro propagation and transcriptional profiling of human mammary stem/progenitor cells. Genes Dev. 2003;17(10):1253-1270.

33. Cabarcas SM, Mathews LA, Farrar WL. The cancer stem cell niche-there goes the neighborhood? Int. J. Cancer. 2011;129:2315-2327.

34. Konopleva MY, Jordan CT. Leukemia stem cells and microenvironment: biology and therapeutic targeting. J Clin Oncol.2011;29:591-599.

35. Mi JK, Young KS. Regulation of Ovarian Cancer Stem Cells or Tumor-Initiating Cells. Int J Mol Sci. 2013;14:6624-6648.

36. Chau WK, Ip CK, Mak AS, et al. c-Kit mediates chemoresistance and tumor-initiating capacity of ovarian cancer cells through activation of Wnt/beta-catenin-ATP-binding cassette G2 signaling. Oncogene. 2012;doi:10.1038/onc.2012.290.

37. Cao L, Shao M, Schilder J, et al. Tissue transglutaminase links TGF-beta, epithelial to mesenchymal transition and a stem cell phenotype in ovarian cancer. Oncogene. 2012;31:2521-2534.

38. Nguyen LV, Vanner R, Dirks P, et al. Cancer stem cells: an evolving concept. Nature Rev. Cancer. 2012;12:133-143.

39. Mitra AK, Zillhardt M, Hua Y, et al. MicroRNAs Reprogram Normal Fibroblasts into Cancer-Associated Fibroblasts in Ovarian Cancer. Cancer Discov. 2012;2:1100-1108.

40. Hanahan D, Weinberg RA. Hallmarks of cancer: The next generation. Cell. 2011;144:646-674.

41. Tirino V, Desiderio V, Paino F, et al. Cancer stem cells in solid tumors: an overview and new approaches for their isolation and characterization. FASEB J. 2013;27(1):13-24.

42. Gaur P, Sceusi EL, Samuel S, et al. Identification of cancer stem cells in human gastrointestinal carcinoid and neuroendocrine tumors. Gastroenterology. 2011;141(5):1728-37.

43. Goodell MA, Brose K, Paradis G, et al. Isolation and functional properties of murine hematopoietic stem cells that are replicating in vivo. J Exp Med. 1996;183:1797-1806.

44. Chiba T, Kita K, Zheng YW, et al. Side population purified from hepatocellular carcinoma cells harbors cancer stem cell-like properties. Hepatology. 2006;44:240-251.

45. Seigel GM, Campbell LM, Narayan M, et al. Cancer stem cell characteristics in retinoblastoma. Mol Vis. 2005;11:729-737.

46. Haraguchi N, Utsunomiya $\mathrm{T}$, Inoue $\mathrm{H}$, et al. Characterization of a side population of cancer cells from human gastrointestinal system. Stem Cells. 2006;24:506-513.

47. Hirschmann-Jax C, Foster AE, Wulf GG, et al. A distinct "side population" of cells with high drug efflux capacity in human tumor cells. Proc Natl Acad Sci. 2004:101:14228-14233.

48. Szotek PP, Pieretti-Vanmarcke R, Masiakos PT, et al. Ovarian cancer side population defines cells with stem cell-like characteristics and Mullerian Inhibiting Substance responsiveness. Proc Natl Acad Sci U S A. 2006;103:11154-11159.

49. Landen CN Jr, Goodman B, Katre AA, et al. Targeting aldehyde dehydrogenase cancer stem cells in ovarian cancer. Mol Cancer Ther. 2010;9(12):3186-3199.

50. Latifi A, Luwor RB, Bilandzic M, et al. Isolation and characterization of tumor cells from the ascites of ovarian cancer patients: molecular phenotype of 
chemoresistant ovarian tumors. PLoS One. 2012;7(10e): 46858. doi: 10.1371/journal.pone.0046858. Epub 2012 Oct 8.

51. Yin AH, Miraglia S, Zanjani ED, et al. AC133, a novel marker for human hematopoietic stem and progenitor cells. Blood. 1997;90:5002-5012.

52. Spangrude GJ, Brooks DM. Mouse strain variability in the expression of the hematopoietic stem cell antigen Ly-6A/E by bone marrow cells. Blood. 1993;82:3327-3332.

53. Moserle L, Indraccolo S, Ghisi M, et al. The side population of ovarian cancer cells is a primary target of IFN-alpha antitumor effects. Cancer Res. 2008;68:5658-5668.

54. Corbeil D, Röper K, Hellwig A, et al. The human AC133 hematopoietic stem cell antigen is also expressed in epithelial cells and targeted to plasma membrane protrusions. J Biol Chem. 2000;275(8):5512-5520.

55. Ferrandina G, Bonanno G, Pierelli L, et al. Expression of CD133-1 and CD133-2 in ovarian cancer. Int J Gynecol Cancer. 2008;18:506-514.

56. Zhang J, Guo X, Chang DY, et al. CD133 expression associated with poor prognosis in ovarian cancer. Mod Pathol. 2012;25:456-464.

57. Baba T, Convery PA, Matsumura N, et al. Epigenetic regulation of CD133 and tumorigenicity of CD133+ ovarian cancer cells. Oncogene. 2009;28(2):209-218.

58. Curley MD, Therrien VA, Cummings CL, et al. CD133 expression defines a tumor initiating cell population in primary human ovarian cancer. Stem Cells. 2009;27(12):2875-83.

59. Heider KH, Kuthan $\mathrm{H}$, Stehle G, et al. CD44v6: a target for antibody-based cancer therapy. Cancer Immunol Immunother. 2004:53:567-579.

60. Misra S, Hascall VC, Berger FG, et al. Hyaluronan, CD44, and cyclooxygenase-2 in colon cancer. Connect Tissue Res. 2008;49(3):219-224.

61. Misra S, Toole BP, Ghatak S. Hyaluronan constitutively regulates activation of multiplereceptor tyrosine kinases in epithelial and carcinoma cells. J Biol Chem. 2006;281(46):34936-34941.

62. Alvero $\mathrm{AB}, \mathrm{Chen} \mathrm{R}, \mathrm{Fu} \mathrm{HH}$, et al. Molecular phenotyping of human ovarian cancer stem cells unravels the mechanisms for repair and chemoresistance. Cell Cycle. 2009;8:158-166.

63. Meng E, Long B, Sullivan P, et al. CD44+/CD24-ovarian cancer cells demonstrate cancer stem cell properties and correlate to survival. Clin Exp Metastasis. 2012;:18.

64. Bapat SA, Mali AM, Koppikar CB, et al. Stem and progenitor-like cells contribute to the aggressive behavior of human epithelial ovarian cancer. Cancer Res. 2005;65:3025-3029.

65. Chen J, Wang J, Chen D, et al. Evaluation of characteristics of CD44 + CD117+ ovarian cancer stem cells in three dimensional basement membrane extract scaffold versus two dimensional monocultures. BMC Cell Biol. 2013;14:7.

66. Wei X, Dombkowski D, Meirelles K, et al. Mullerian inhibiting substance preferentially inhibits stem/progenitors in human ovarian cancer cell lines compared with chemotherapeutics. Proc Natl Acad Sci USA. 2010;107(44):18874-9.

67. Zhang S, Balch C, Chan MW, et al. Identification and characterization of ovarian cancer-initiating cells from primary human tumors. Cancer Res. 2008:68:4311-4320.

68. Alvero $\mathrm{AB}, \mathrm{Chen} \mathrm{R}, \mathrm{Fu} \mathrm{HH}$, et al. Molecular phenotyping of human ovarian cancer stem cells unravel the mechanisms for repair and chemo-resistance. Cell Cycle. 2009;8(Suppl. 1):158-166.

69. Kristiansen G, Sammar M, Altevogt P. Tumour biological aspects of CD24, a mucin-like adhesion molecule. J Mol Histol. 2004;35(3):255-262.

70. Surowiak P, Materna V, Kaplenko I, et al. Unfavorable prognostic value of $\mathrm{CD} 24$ expression in sections from primary and relapsed ovarian cancer tissue. International Journal of Gynecological Cancer. 2006;16(2):515-521.

71. Choi YL, Kim SH, Shin YK, et al. Cytoplasmic CD24 expression in advanced ovarian serous borderline tumors. Gynecologic Oncology. 2005;97(2):379-386.

72. Gao MQ, Choi YP, Kang S, et al. CD24+ cells from hierarchically organized ovarian cancer are enriched in cancer stem cells. Oncogene. 2010;29(18):2672-2680.

73. Luo L, Zeng J, Liang B, et al. Ovarian cancer cells with the CD117 phenotype are highly tumorigenic and are related to chemotherapy outcome. Exp Mol Pathol. 2011;91:596-602.

74. Raspollini MR, Amunni G, Villanucci A, et al. c-KIT expression and correlation with chemotherapy resistance in ovarian carcinoma: an immunocytochemical study. Ann Oncol. 2004;15(4):594-597.

75. Imrich S, Hachmeister M, Gires O. EpCAM and its potential role in tumor-initiating cells. Cell Adh Migr. 2012;6:30-38

76. Bellone S, Siegel ER, Cocco E, et al. Overexpression of epithelial cell adhesion molecule in primary, metastatic, and recurrent/chemotherapyresistant epithelial ovarian cancer: Implications for epithelial cell adhesion molecule-specific immunotherapy. Int J Gynecol Cancer. 2009;19:860-866.

77. Yamashita T, Ji J, Budhu A, et al. EpCAM-positive hepatocellular carcinoma cells are tumor initiating cells with stem/progenitor cell features. Gastroenterology. 2009;136:1012-1024.

78. Lawrenson K, Gayther SA. Ovarian cancer: a clinical challenge that needs some basic answers. PLoS Med 2009; 6:e25.

79. Tothill IE. Biosensors for cancer markers diagnosis. Semin Cell Dev Biol. 2009;20:55-62.

80. Liu P, Brown S, Goktug T, et al. Cytotoxic effect of disulfiram/copper on human glioblastoma cell lines and ALDH-positive cancer-stem-like cells. Br J of Cancer.2012;107:1488-1497.
81. Croker AK, Goodale D, Chu J, et al. High aldehyde dehydrogenase and expression of cancer stem cell markers selects for breast cancer cells with enhanced malignant and metastatic ability. J Cell Mol Med. 2009;13:2236-2252.

82. Silva IA, Bai S, McLean K, et al. Aldehyde dehydrogenase and CD133 define angiogenic ovarian cancer stem cells that portend poor patient survival. Cancer Res. 2011;71:3991-4001.

83. Ginestier $\mathrm{C}$, Hur MH, Charafe-Jauffret E, et al. ALDH1 is a marker of normal and malignant human mammary stem cells and a predictor of poor clinical outcome. Cell Stem Cell. 2007;1:555-567.

84. García Campelo MRACG, Aparicio Gallego G, Grande Pulido E, et al. Stem cell and lung cancer development: Blaming the Wnt, Hh and Notch signalling pathway. Clin Transl Oncol. 2011;13:77-83.

85. Rask K, Nilsson A, Brannstrom M, et al. Wnt-signalling pathway in ovarian epithelial tumours: Increased expression of beta-catenin and GSK3beta. Br J Cancer. 2003;89:1298-1304.

86. Morris RJ, Liu Y, Marles L, et al. Capturing and profiling adult hair follicle stem cells. Nat Biotechnol. 2004;22:411-417.

87. McClanahan T, Koseoglu S, Smith K, et al. Identification of overexpression of orphan G protein-coupled receptor GPR49 in human colon and ovarian primary tumors. Cancer Biol Ther. 2006;5:419-426.

88. Colomiere M, Ward AC, Riley C, et al. Cross talk of signals between EGFR and IL-6R through JAK2/STAT3 mediate epithelial-mesenchymal transition in ovarian carcinomas. Br J Cancer. 2009;100:134-144.

89. Steg AD, Katre AA, Goodman B, et al. Targeting the notch ligand JAGGED1 in both tumor cells and stroma in ovarian cancer. Clin Cancer Res. 2011;17:5674-5685

90. Reya T, Morrison SJ, Clarke MF, et al. Stem cells, cancer, and cancer stem cells. Nature. 2001;414:105-111

91. Tomao F, Papa A, Rossi L, et al. Current status of bevacizumab in advanced ovarian cancer. Onco Targets Ther. 2013;6:889-99. doi: 10.2147/OTT.S46301.

92. Ishii $\mathrm{H}$, Iwatsuki M, Ieta $\mathrm{K}$, et al. Cancer stem cells and chemoradiation resistance. Cancer Sci. 2008;99:1871-1877.

93. Gimenez-Bonafe P, Tortosa A, Perez-Tomas R. Overcoming drug resistance by enhancing apoptosis of tumor cells. Curr Cancer Drug Targets. 2009;9:320-340.

94. Jordan CT, Guzman ML, Noble M. Cancer stem cells. N Engl J Med. 2006;355:1253-1261.

95. Dean M. ABC transporters, drug resistance, and cancer stem cells. J Mammary Gland Biol Neoplasia. 2009:14:3-9.

96. Donnenberg VS, Meyer EM, Donnenberg AD. Measurement of multiple drug resistance transporter activity in putative cancer stem/progenitor cells. Methods Mol Biol. 2009;568:261-279.

97. Anderson ME. Glutathione: an overview of biosynthesis and modulation. Chem Biol Interact. 1998;111-112:1-14.

98. Backos DS, Franklin CC, et al. The role of glutathione in brain tumor drug resistance. Biochem Pharmacol. 2012;83(8):1005-1012.

99. Wu WJ, Zhang $\mathrm{Y}$, Zeng $\mathrm{ZL}$, et al. $\beta$-phenylethyl isothiocyanate reverses platinum resistance by a GSH-dependent mechanism in cancer cells with epithelial-mesenchymal transition phenotype. Biochem Pharmacol. 2013;85(4):486-96.

100. Kusumbe AP, Bapat SA. Cancer stem cells and aneuploid populations within developing tumors are the major determinants of tumor dormancy. Cancer Res. 2009:69:9245-9253.

101. Peinado H, Portillo F, Cano A. Transcriptional regulation of cadherins during development and carcinogenesis. Int J Dev Biol. 2004;48:365-375.

102. Polyak K, Weinberg RA. Transitions between epithelial and mesenchymal states: acquisition of malignant and stem cell traits. Nat Rev Cancer. 2009;9:265-273.

103. Wicha MS, Liu S, Dontu G. Cancer stem cells: an old idea-a paradigm shift. Cancer Res. 2006;66:1883-1890.

104. Sell S, Pierce GB. Maturation arrest of stem cell differentiation is a common pathway for the cellular origin of teratocarcinomas and epithelial cancers. Lab Invest. 1994;70:6-22.

105. Yo YT, Lin YW, Wang YC, et al. Growth inhibition of ovarian tumor-initiating cells by niclosamide. Mol. Cancer Ther. 2012;11:1703-1712.

106. Alvero AB, Montagna MK, Holmberg JC, et al. Targeting the mitochondria activates two independent cell death pathways in ovarian cancer stem cells. Am J Reprod Immunol. 2011;65:438-447.

107. Leizer AL, Alvero $\mathrm{AB}, \mathrm{Fu} \mathrm{HH}$, et al. Regulation of inflammation by the NF-kappaB pathway in ovarian cancer stem cells. Mol Cancer Ther. 2011;10:1385-1393.

108. Shank JJ, Yang K., Ghannam J, et al. Metformin targets ovarian cancer stem cells in vitro and in vivo. Gynecol Oncol 2012;127:390-397.

109. Slomiany MG; Dai L, Tolliver LB, et al. Inhibition of Functional Hyaluronan-CD44 Interactions in CD133-positive Primary Human Ovarian Carcinoma Cells by Small Hyaluronan Oligosaccharides. Clin Cancer Res. 2009;15:7593-7601.

110. Casagrande F, Cocco E, Bellone S, et al. Eradication of chemotherapy-resistant CD44+ human ovarian cancer stem cells in mice by intraperitoneal administration of Clostridium perfringens enterotoxin. Cancer. 2011;117:5519-5528.

111. McAuliffe SM, Morgan SL, Wyant GA, et al. Targeting Notch, a key pathway for ovarian cancer stem cells, sensitizes tumors to platinum therapy. Proc Natl Acad SciUSA. 2012;109 (E): 2939-2948.

112. Jin L., Hope KJ., Zhai Q., et al. Targeting of CD44 eradicates human acute myeloid leukemic stem cells. Nature Med. 2006;12:1167-1174. 
113. Vici P, Sergi D, Pizzuti L, et al. Gemcitabine-oxaliplatin (GEMOX) as salvage treatment in pretreated epithelial ovarian cancer patients. J Exp Clin Cancer Res. 2013;32(1):49

114. Van Rhenen A, van Dongen GAMS, Kelder A, et al. The novel AML stem cell associated antigen CLL1 aids in discrimination between normal and leukemic stem cells. Blood. 2007;110:2659-2666.

115. Jin L, Lee EM, Ramshaw HS, et al. Monoclonal antibody-mediated targeting of CD123, IL3 receptor a chain, eliminates human acute myeloid leukemic stem cells. Cell Stem Cell. 2009;5:31-42.

116. Jores $\mathrm{M}$, Johnels $\mathrm{P}$, Hansen $\mathrm{N}$, et al. Isolation and killing of candidate chronic myeloid leukemia stem cells by antibody targeting of IL1 receptor accessory protein. Proc Natl Acad Sci USA 2010;107:16280-16285.

117. Tomao F, Panici PB, Frati L, et al. Emerging role of pemetrexed in ovarian cancer. Expert Rev Anticancer Ther. 2009;9(12):1727-35. doi: 10.1586/era.09.141.

118. Tomao F, Papa A, Rossi L, et al. Beyond bevacizumab: investigating new angiogenesis inhibitors in ovarian cancer. Expert Opin Investig Drugs. 2014;23(1):37-53. doi: 10.1517/13543784.2013.839657.

119. Korkaya H, Wicha MS. Selective targeting of cancer stem cells: a new concept in cancer therapeutics. BioDrugs 2007;21:299-310.

120. Tu LC, Foltz G, Lin E, et al. Targeting stem cells-clinical implications for cancer therapy. Curr Stem Cell Res Ther. 2009;4:147-153.

121. Gupta PB, Onder TT, Jiang G, et al. Identification of selective inhibitors of cancer stem cells by high-throughput screening. Cell. 2009;138:645-659.

122. Curtin JC, Lorenzi MV. Drug discovery approaches to target Wnt signaling in cancer stem cells. Oncotarget. 2010;1:563-577.

123. Tomao F, Tomao S, Panici PB. Improvement in progression-free survival in OCEANS bevacizumab arm: a critical point of view. J Clin Oncol. 2013;31(1):166-7. doi: 10.1200/JCO.2012.44.6237.

124. Martelli AM, Evangelisti C, Follo YM, et al. Targeting the phosphatidylinositol 3kinase/Akt/mammalian target of rapamycin signaling network in cancer stem cells. Curr Med Chem. 2011;18:2715-2726.

125. Takebe N, Harris PJ, Warren RQ, et al. Targeting cancer stem cells by inhibiting Wnt, Notch, and Hedgehog pathways. Nature Rev Clin Oncol. 2011;8:97-106 